\title{
IMPLICAÇÕES SOCIAIS E DE SAÚDE QUE ACOMETEM PESSOAS ENLUTADAS PELA MORTE DE FAMILIARES POR COVID-19
}

\author{
SOCIAL AND HEALTH IMPLICATIONS THAT \\ AFFECT PEOPLE BEREAVED BY THE DEATH \\ OF FAMILY MEMBERS BY COVID-19
}

\section{IMPLICACIONES SOCIALES Y DE SALUD QUE AFECTAN LAS PERSONAS EN LUTO POR LA MUERTE DE FAMILIARES POR LA COVID-19}

\author{
Julia Renata Fernandes de Magalhães ${ }^{1}$ \\ Caroline Fernandes Soares e Soares ${ }^{2}$ \\ Thaís Moreira Peixoto ${ }^{3}$ \\ Fernanda Matheus Estrela ${ }^{4}$ \\ Ana Carla Barbosa de Oliveira ${ }^{5}$ \\ Andrey Ferreira da Silva ${ }^{6}$ \\ Nadirlene Pereira Gomes
}

Como citar este artigo: Magalhães JRF, Soares CFS, Peixoto TM, Estrela FM, Oliveira ACB, Silva AF, et al. Implicações Sociais e de Saúde que acometem pessoas enlutadas pela morte de familiares por COVID-19. Rev baiana enferm. 2020;34:e37007.

Objetivo: conhecer as implicações sociais e para a saúde que acometem pessoas enlutadas pela morte de familiares vítimas da COVID-19. Método: trata-se de revisão narrativa realizada na plataforma PubCovid-19. Resultados: foi desenvolvido um quadro apresentando as características das dez publicações segundo título, autoria, ano de publicação, país e periódico. Conclusão: as implicações sociais e de saúde que acometem pessoas enlutadas pela morte de familiares por COVID-19 decorrem do distanciamento social que gera a impossibilidade da realização de rituais de despedida prejudicando a vivência normal do luto, levando ao luto complicado e ao possível adoecimento psíquico.

Descritores: Morte. Luto. Covid-19. Saúde Mental. Isolamento.

Objective: to know the social and health implications that affect people bereaved by the death of relatives who were victims of COVID-19. Method: this is a narrative review carried out on the PubCovid-19 platform. Results: a chart was developed presenting the characteristics of the ten publications by title, authorship, year of publication, country and

\footnotetext{
Enfermeira. Mestre em Enfermagem. Universidade Federal da Bahia. Guanambi, Bahia, Brasil. https://orcid.org/0000-0003-063 I-2374

Estudante de Enfermagem. Universidade Estadual de Feira de Santana. Feira de Santana, Bahia, Brasil. https://orcid.org/0000-0003-4464-8389.

Enfermeira. Mestre em Saúde Coletiva. Professora Substituta da Universidade Estadual de Feira de Santana. Feira de Santana, Bahia, Brasil. https://orcid.org/0000000I-5395-0905.

4 Enfermeira. Doutora em Enfermagem. Professora da Universidade Estadual de Feira de Santana. Feira de Santana, Bahia, Brasil. nanmatheus@yahoo.com.br. https://orcid.org/0000-000 I-750 I-6187.

Enfermeira. Universidade Estadual de Feira de Santana. Feira de Santana, Bahia, Brasil. https://orcid.org/0000-0002-I I 75-8074

Enfermeiro. Doutor em Enfermagem. Professor da Universidade Federal de Alagoas. Maceió, Alagoas, Brasil. http://orcid.org/0000-0002-1038-7443.

Enfermeira. Doutora em Enfermagem. Professora da Universidade Federal da Bahia. Salvador, Bahia, Brasil. http://orcid.org/0000-0002-6043-3997.
} 
journal. Conclusion: the social and health implications that affect people bereaved by the death of family members by COVID-19 derive from the social distancing that generates the impossibility of performing farewell rituals, damaging the normal experience of mourning, leading to complicated mourning and possible psychic illness.

Descriptors: Death. Mourning. Covid-19. Mental Health. Isolation.

Objetivo: conocer las implicaciones sociales y para la salud que afectan a las personas en luto por la muerte de familiares víctimas de COVID-19. Método: esta es una revisión narrativa realizada en la plataforma PubCovid-19. Resultados: se elaboró un cuadro que presenta las características de las diez publicaciones por título, autoría, año de publicación, país y periódico. Conclusión: las implicaciones sociales y para la salud que afectan a las personas en luto por la muerte de familiares por la COVID-19 se derivan del distanciamiento social que genera la imposibilidad de realizar los rituales de despedida, perjudicando la vivencia normal del luto, provocando el complicado luto y la posible enfermedad psíquica.

Descriptores: La muerte. Luto. Covid-19. Salud Mental. Aislamiento.

\section{Introdução}

A pandemia da COVID-19, causada pelo SARS-CoV-2, tem se propagado de forma devastadora, causando diversos impactos na sociedade relacionados à alta transmissibilidade do vírus e elevados índices de mortalidade. Dessa forma, o Ministério da Saúde (MS) publicou um guia de manejo de corpos no contexto do novo coronavírus $^{(1)}$, com recomendações acerca da não realização de funerais, o que reverbera no processo de luto sem despedida, causando repercussões sociais e para a saúde na vida dos familiares enlutados.

Os primeiros casos da doença no mundo foram notificados em uma província da China, em 31 de dezembro de 2019, sendo declarada como uma pandemia pela Organização Mundial de Saúde (OMS) em março de 2020. Estudos internacionais e nacionais revelam que até o dia 22 de maio de 2020 ocorreram 330 mil óbitos por complicações da doença ${ }^{(2)}$. No Brasil, o número de mortos é superior a 21 mil, o que coloca o país na $3^{\underline{a}}$ posição em mortes, com 6,4\% de letalidade ${ }^{(3)}$.

Diante da rápida elevação do número de óbitos, as autoridades sanitárias instituíram alterações nos formatos de velórios e sepultamentos durante a pandemia, determinando a proibição de velório para os casos confirmados da COVID-19, de forma que a urna deve sair do carro da funerária direto para a sepultura ${ }^{(1)}$.
Os rituais de despedidas adotados diante da morte de um familiar facilitam o processo de elaboração, ainda que relativa, dos sentimentos que emergem nesse contexto, facilitando a adoção de estratégias de enfrentamento desse processo ${ }^{(4-5)}$.

O contexto da pandemia exigiu que os familiares enlutados por vitimados pela COVID-19 tivessem que se adaptar à impossibilidade de se despedir de seus entes queridos, o que pode repercutir no contexto social e na saúde dessa população. Diante disso, levantamos a seguinte questão de pesquisa: Quais as implicações sociais e para esses familiares em luto pela COVID-19?

Este estudo tem por objetivo conhecer as implicações sociais e para a saúde que acometem pessoas enlutadas pela morte de familiares vítimas da COVID-19.

\section{Método}

Este estudo caracteriza-se como revisão narrativa da literatura, de forma que o corpus de análise é formado pelo referencial teórico dos artigos levantados, e seus resultados possibilitam evidenciar novas reflexões e ampliar o conhecimento sobre o luto de familiares vitimados pela COVID-19 ${ }^{(6)}$. Ressalta-se que a seleção dos estudos pode estar sujeita à subjetividade do autor.

A busca dos artigos ocorreu no mês de maio de 2020, na plataforma PubCovid-19, a qual está 
indexada na Biblioteca Nacional de Medicina dos Estados Unidos (PUBMED) e no Banco de dados Excerpta Medica (EMBASE). A plataforma PubCovid-19 foi criada com o objetivo de reunir as publicações relacionadas à pandemia pela COVID-19 e organizar os artigos por áreas temáticas a fim de possibilitar o acesso aos pesquisadores. Para a realização da busca foram utilizados os seguintes descritores em inglês: "Death"; "Bereavement"; "Grief" e "Mourning", visando à delimitação temática.

Foram incluídos artigos científicos que refletiam sobre a COVID-19 e faziam interface com as temáticas: mortes pela COVID-19, luto, implicações sociais e para a saúde de pessoas enlutadas.

Foram selecionados inicialmente 84 artigos. Após leitura dos títulos e resumos, 74 foram excluídos por não abordarem o tema delimitado, restando 10 artigos para leitura na íntegra. Para melhor catalogação, foi utilizado um instrumento criado pelos pesquisadores para compilar as publicações, contendo as seguintes informações: título, autoria, ano de publicação, país, periódico, metodologia adotada no estudo e as implicações sociais e para a saúde que acometem pessoas enlutadas diante da morte de familiares pela COVID-19.

Os estudos foram lidos exaustivamente, categorizados e analisados com vistas à busca das implicações. Após a exploração do material selecionado, a discussão foi desenvolvida considerando as categorias pré-definidas: Implicações sociais e Implicações para a saúde.

\section{Resultados}

Com base nas dez publicações selecionadas, foi desenvolvido um quadro com as características dessas publicações, segundo título, autoria, ano de publicação, periódico, país, tipo de estudo e implicações sociais e para a saúde (Quadro1).

Quadro 1 - Síntese dos estudos selecionados na base de dados PubCovid-19, por título, autor, ano, publicação, país, tipo de estudo e implicações

(continua)

\begin{tabular}{|c|c|c|c|c|}
\hline $\mathbf{N}^{\circ}$ & Título & Autor, Ano, Publicação, País & $\begin{array}{c}\text { Tipo de } \\
\text { estudo }\end{array}$ & $\begin{array}{c}\text { Implicações sociais e } \\
\text { para a saúde }\end{array}$ \\
\hline $\mathbf{1}$ & $\begin{array}{l}\text { Pursuing a Good } \\
\text { Death in the Time } \\
\text { of COVID-19 }\end{array}$ & $\begin{array}{l}\text { Wang SSY, Teo WZY, Yee CW, } \\
\text { Chai YW }(2020)^{7} \\
\text { Journal of Palliative Medicine } \\
\text { Singapura }\end{array}$ & Reflexão & $\begin{array}{l}\text { Sociais: não realização } \\
\text { dos velórios, ausência } \\
\text { de afeto e abraços. } \\
\text { Para a saúde: } \\
\text { sentimentos de } \\
\text { ansiedade e angústia. }\end{array}$ \\
\hline 2 & $\begin{array}{l}\text { Death in the era } \\
\text { of the COVID-19 } \\
\text { pandemic }\end{array}$ & $\begin{array}{l}\text { Ingravallo, F (2020) } \\
\text { The Lancet Public Health } \\
\text { Estados Unidos da América }\end{array}$ & Reflexão & $\begin{array}{l}\text { Sociais: mudança } \\
\text { na forma como são } \\
\text { realizados os rituais } \\
\text { fúnebres. }\end{array}$ \\
\hline 3 & $\begin{array}{l}\text { COVID-19 and } \\
\text { Unfinished } \\
\text { Mourning }\end{array}$ & $\begin{array}{l}\text { Farahmandnia B, Hamdanieh L, } \\
\text { Aghababaeian H (2020) } \\
\text { Prehospital and Disaster } \\
\text { Medicine } \\
\text { Irã }\end{array}$ & Reflexão & $\begin{array}{l}\text { Para a saúde: } \\
\text { adoecimento mental. }\end{array}$ \\
\hline 4 & $\begin{array}{l}\text { Mourning } \\
\text { our dead in } \\
\text { the covid-19 } \\
\text { pandemic }\end{array}$ & $\begin{array}{l}\text { O'Mahony, S }(2020)^{10} \\
\text { British Medical Journals } \\
\text { Inglaterra }\end{array}$ & Reflexão & $\begin{array}{l}\text { Para a saúde: sofrimento } \\
\text { patológico e depressão. }\end{array}$ \\
\hline 5 & $\begin{array}{l}\text { Loss and grief } \\
\text { amidst COVID-19: } \\
\text { A path to } \\
\text { adaptation and } \\
\text { resilience }\end{array}$ & $\begin{array}{l}\text { Zhai Y, Du X (2020) } \\
\text { Brain, Behavior, and Immunity } \\
\text { Estados Unidos da América }\end{array}$ & Reflexão & $\begin{array}{l}\text { Sociais: quebra de laços } \\
\text { afetivos e parentais. } \\
\text { Para a saúde: sofrimento } \\
\text { psíquico associado à } \\
\text { perda. }\end{array}$ \\
\hline
\end{tabular}


Quadro 1 - Síntese dos estudos selecionados na base de dados PubCovid-19, por título, autor, ano, publicação, país, tipo de estudo e implicações

(conclusão)

\begin{tabular}{|c|c|c|c|c|}
\hline $\mathbf{N}^{\circ}$ & Título & Autor, Ano, Publicação, País & $\begin{array}{l}\text { Tipo de } \\
\text { estudo }\end{array}$ & $\begin{array}{c}\text { Implicações sociais e } \\
\text { para a saúde }\end{array}$ \\
\hline 6 & $\begin{array}{l}\text { Grief Duringthe } \\
\text { COVID-19 } \\
\text { Pandemic: } \\
\text { Considerations } \\
\text { for Palliative Care } \\
\text { Providers }\end{array}$ & $\begin{array}{l}\text { Wallace CL, Wladkowski SP, } \\
\text { Gibson A, White P }(2020)^{12} \\
\text { Journal of Pain and Symptom } \\
\text { Management } \\
\text { Estados Unidos da América }\end{array}$ & Reflexão & $\begin{array}{l}\text { Sociais: apoio social } \\
\text { reduzido e não } \\
\text { compartilhamento do } \\
\text { sofrimento. } \\
\text { Para a saúde: sofrimento } \\
\text { mental acentuado. }\end{array}$ \\
\hline 7 & $\begin{array}{l}\text { Addressing } \\
\text { mental health } \\
\text { care for the } \\
\text { bereaved during } \\
\text { the COVID-19 } \\
\text { pandemic }\end{array}$ & $\begin{array}{l}\text { Sun Y, Bao Y, Lu L }(2020)^{13} \\
\text { Psychiatry and Clinical } \\
\text { Neurosciences } \\
\text { China }\end{array}$ & Reflexão & $\begin{array}{l}\text { Sociais: separação dos } \\
\text { membros da família. } \\
\text { Para a saúde: estado } \\
\text { de luto patológico } \\
\text { (tristeza, angústia, culpa, } \\
\text { depressão, transtorno de } \\
\text { estresse pós-traumático). }\end{array}$ \\
\hline 8 & $\begin{array}{l}\text { Supporting adults } \\
\text { bereaved through } \\
\text { COVID-19: a } \\
\text { rapid review } \\
\text { of the impact } \\
\text { of previous } \\
\text { pandemics } \\
\text { on grief and } \\
\text { bereavement }\end{array}$ & $\begin{array}{l}\text { Mayland CR, Harding AJ, } \\
\text { Preston N, Payne S }(2020)^{14} \\
\text { Journal of Pain and Symptom } \\
\text { Management } \\
\text { Reino Unido }\end{array}$ & Revisão & $\begin{array}{l}\text { Sociais: mudança nas } \\
\text { normas sociais e rituais } \\
\text { de luto. } \\
\text { Para a saúde: risco de } \\
\text { luto patológico. }\end{array}$ \\
\hline 9 & $\begin{array}{l}\text { Bereavement } \\
\text { in the Time of } \\
\text { Coronavirus: } \\
\text { Unprecedented } \\
\text { Challenges } \\
\text { Demand Novel } \\
\text { Interventions }\end{array}$ & $\begin{array}{l}\text { Carr D, Boerner K, Moorman S } \\
(2020)^{15} \\
\text { Journal of Aging \& Social Policy } \\
\text { Estados Uniddos da América }\end{array}$ & Reflexão & $\begin{array}{l}\text { Para a saúde: tristeza, } \\
\text { angústia, dor, culpa e } \\
\text { raiva. }\end{array}$ \\
\hline 10 & $\begin{array}{l}\text { Supporting } \\
\text { families in end- } \\
\text { of-life care and } \\
\text { bereavement in } \\
\text { the COVID-19 }\end{array}$ & $\begin{array}{l}\text { Moore K , Sampson EL, Kupeli N, } \\
\text { Davies N }(2020)^{16} \\
\text { International Psycho Geriatrics } \\
\text { United Kingdom }\end{array}$ & Reflexão & $\begin{array}{l}\text { Sociais: restrição de } \\
\text { visitas de familiares e } \\
\text { amigos. } \\
\text { Para a saúde: tristeza } \\
\text { profunda e depressão. }\end{array}$ \\
\hline
\end{tabular}

Fonte: Elaboração própria.

\section{Discussão}

\section{Implicações sociais}

De acordo com a literatura científica pesquisada, a impossibilidade da realização de rituais de despedida de familiares mortos pela COVID-19 tem desencadeado importantes implicações sociais, dentre elas, destaca-se a frustração por não poder proporcionar um funeral considerado digno para o ente falecido ${ }^{(6)}$. No que diz respeito aos significados presentes nesses rituais, considera-se que estes representam a oportunidade para os enlutados expressarem os sentimentos e as emoções sobre os entes perdidos ${ }^{(8)}$, possibilitando a manifestação pública do pesar e a criação de um momento de comunhão, cumplicidade e compaixão ${ }^{(17)}$.

As formas de velar e se despedir dos mortos variam conforme a cultura. No Brasil, por exemplo, os funerais costumam ser realizados no prazo médio de dois dias, já na Alemanha e Estados Unidos da América, podem durar até uma 
semana $^{(18)}$. Com a disseminação da COVID-19, essas manifestações têm sido bruscamente rompidas, fazendo com que os familiares necessitem buscar caminhos para lidar sozinhos com o fenômeno da morte e com a sobrecarga emocional gerada por esse evento ${ }^{(6)}$.

Sob esse olhar, os estudos analisados demonstram que as mortes relacionadas a COVID-19 têm sido socialmente compreendidas como desprovidas de dignidade e como uma "morte ruim". Esta percepção está atrelada aos sentimentos de dor pelo sofrimento solitário do familiar, de culpa por não poder protegê-lo, e de injustiça, por acreditarem que os tratamentos foram ineficazes.

É válido ressaltar que a visão acerca da morte vem sofrendo transformações ao longo da história, passando de uma experiência que era lidada com naturalidade, como ocorria na Idade Média, para se tornar algo com o qual todos buscam se afastar, causador de angústia, dor e aflição ${ }^{(19)}$. Em meio a esse processo doloroso de perda, o luto se manifesta como um conjunto de reações emocionais, físicas, cognitivas e comportamentais expressas, dentre outras formas, pela tristeza, solidão, culpa, ansiedade e preocupação $^{(17)}$. Todas essas reações são manifestadas como vivência do luto.

Em alguns casos, quando ocorre um contexto de doenças terminais com alta probabilidade de morte, os familiares, para lidar com a perda anunciada, passam a trabalhar para enfrentar a perda de modo antecipado - o denominado luto antecipatório, que se manifesta antes mesmo do paciente evoluir com complicações graves da doença ${ }^{(5)}$.

Ainda, cabe salientar que nos casos de COVID-19 existe um estigma que permeia a vida da pessoa doente, devido às incertezas geradas pelo vírus e o enorme aumento de mortes.

A rede de apoio formada por familiares e amigos representa um suporte essencial para o enfrentamento desse fenômeno, já que pessoas enlutadas tendem a se sentir aliviadas ao receber um abraço, ao ter um ombro para chorar ou ao compartilhar os sentimentos com alguém ${ }^{(19)}$. Com o contexto do distanciamento social imposto pela pandemia, contudo, as pessoas têm sido privadas desse acolhimento, o que reverbera na intensificação e prolongamento das manifestações clínicas, fazendo com o que o luto deixe de ser uma vivência natural para se tornar complicado ou até mesmo patológico ${ }^{(8,11-12)}$.

\section{Implicações para a saúde}

No que se refere ao processo de adoecimento, os estudos evidenciam que as pessoas que perderam familiares por COVID-19, que não puderam receber o conforto dos parentes ou não tiveram a chance de se despedir dos entes falecidos, são mais propícias a vivenciar o luto complicado e até patológico, além de apresentar outros comprometimentos psicológicos, dentre eles o humor depressivo, o transtorno de estresse pós-traumático, a preocupação exagerada, a angústia, a dificuldade em aceitar a morte e o desinteresse pela vida ${ }^{(9-10,12-13)}$.

Os especialistas chegam a prever, inclusive, uma epidemia tardia de luto patológico após o término da pandemia da COVID-19, tendo em vista as estatísticas alarmantes de óbitos por essa causa e a ausência de estratégias de cuidado, sendo necessária a inclusão de um cuidado intersetorial fornecendo reabilitação, programas e aconselhamento especializado à família e parentes do falecido e garantindo um acompanhamento contínuo ${ }^{(9-10,14-16)}$. Todos esses comprometimentos para a vida e a saúde de pessoas que perderam familiares por COVID-19 demonstram a urgência de medidas que as auxiliem a responderem adequadamente ao luto, na tentativa de minimizar os impactos imediatos e tardios decorrentes da pandemia.

\section{Conclusão}

Os estudos analisados evidenciaram que as implicações sociais e de saúde que acometem pessoas enlutadas pela morte de familiares por COVID-19 decorrem da impossibilidade da realização de rituais de despedida, por conta do distanciamento social, fatores que prejudicam a vivência do luto, levando ao adoecimento psíquico manifestado, dentre outras formas, pela 
depressão, transtorno do estresse pós-traumático, preocupação excessiva, angústia e desinteresse pela vida.

Tendo em vista a tendência de esses efeitos perdurarem pós-pandemia, urge a necessidade da ampliação da rede de atenção psicossocial para atuarem com estratégias de suporte emocional, não apenas de forma imediata, como também em longo prazo.

Dentre as estratégias de suporte emocional, sugere-se: adoção de técnicas remotas de despedida, a exemplo das chamadas de vídeo; fortalecimento das redes religiosas e/ou espirituais; além da humanização no processo de comunicação do óbito. Ressalta-se a necessidade do aprofundamento de estudos relacionados ao cuidado às pessoas enlutadas pela morte de familiares por COVID-19, tendo em vista que as pesquisas estão voltadas, em sua maioria, para os aspectos epidemiológicos da doença.

\section{Colaborações:}

1 - concepção, projeto, análise e interpretação dos dados: Julia Renata Fernandes de Magalhães, Caroline Fernandes Soares e Soares, Thaís Moreira Peixoto, Fernanda Matheus Estrela, Ana Carla Barbosa de Oliveira e Andrey Ferreira da Silva;

2 - redação do artigo e revisão crítica relevante do conteúdo intelectual: Julia Renata Fernandes de Magalhães, Caroline Fernandes Soares e Soares, Thaís Moreira Peixoto, Fernanda Matheus Estrela, Ana Carla Barbosa de Oliveira, Andrey Ferreira da Silva e Nadirlene Pereira Gomes;

3 - aprovação final da versão a ser publicada: Julia Renata Fernandes de Magalhães, Caroline Fernandes Soares e Soares, Thaís Moreira Peixoto, Fernanda Matheus Estrela, Ana Carla Barbosa de Oliveira, Andrey Ferreira da Silva e Nadirlene Pereira Gomes.

\section{Referências}

1. Brasil. Ministério da Saúde. Manejo de corpos no contexto do novo coronavírus Covid-19 [Internet].
Brasília (DF); 2020 [cited 2020 May 23]. Available from: https://portalarquivos.saude.gov.br/images/ pdf/2020/marco/25/manejo-corpos-coronavirusversao1-25mar20-rev5.pdf

2. Freitas ARR, Napimoga M, Donalisio MR. Análise da gravidade da pandemia de Covid-19. Epidemiol Serv Saúde. 2020;29(2):e2020119. DOI: https://doi. org/10.5123/s1679-49742020000200008

3. Brasil. Ministério da Saúde. Painel Coronavírus [Internet]. Brasília (DF); 2020 [cited 2020 May 23]. Available from: https://covid.saude.gov.br/

4. Brasil. Ministério da Saúde. Manejo de corpos no contexto do novo coronavírus Covid-19 [Internet]. Brasília (DF); 2020 [cited 2020 May 23]. Available from: https://portalarquivos.saude.gov.br/images/ pdf/2020/marco/25/manejo-corpos-coronavirusversao1-25mar20-rev5.pdf

5. Ondere Neto J, Lisboa CSM. Doenças associadas ao luto antecipatório: uma revisão da literatura. Psic., Saúde \& Doenças. 2017 ago;18(2):308-21. DOI: http://dx.doi.org/10.15309/17psd180203

6. Toledo JÁ, Rodrigues MC. Teoria da mente em adultos: uma revisão narrativa da literatura. Bol - Acad Paul Psicol [Internet]. 2017 [cited 2020 May 19];37(92):139-56. Available from: http://pepsic.bvsalud.org/scielo.php?script=sci_ arttext\&pid $=$ S1 1415-711X2017000100011\&lng=pt\& $\mathrm{nrm}=\mathrm{iso}$

7. Wang SSY, Teo WZY, Yee CW, Chai YW. Pursuing a Good Death in the Time of COVID-19. J Palliat Med. 2020;23(6). DOI: 10.1089/ jpm.2020.0198

8. Ingravallo $\mathrm{F}$. Death in the era of the COVID-19 pandemic. Lancet Public Health. 2020;5(5):e258. DOI: $10.1016 /$ S2468-2667(20)30079-7

9. Farahmandnia B, Hamdanieh L, Aghababaeian H. COVID-19 and Unfinished Mourning. Prehosp Disaster Med. 2020;1. DOI: 10.1017/ S1049023X20000631

10. O'Mahony S. Mourningourdead in the covid-19 pandemic. BMJ. 2020 Apr 27;369:m1649. DOI: 10.1136/bmj.m1649

11. Zhai Y, Du X. Loss and grief amidst COVID-19: A path to adaptation and resilience. Brain Behav Immun. 2020 Apr 23. DOI: 10.1016/ j.bbi.2020.04.053

12. Wallace CL, Wladkowski SP, Gibson A, White P. Grief During the COVID-19 Pandemic: Considerations for Palliative Care Providers. 
Julia Renata Fernandes de Magalhães, Caroline Fernandes Soares e Soares, Thaís Moreira Peixoto,' Fernanda Matheus Estrela, Ana Carla Barbosa de Oliveira, Andrey Ferreira da Silva, Nadirlene Pereira Gomes

J Pain Symptom Manage. 2020 Apr 13. DOI: 10.1016/ j.jpainsymman.2020.04.012

13. Sun Y, Bao Y, Lu L. Addressing mental healthcare for the bereaved during the COVID-19 pandemic. Psychiatry Clin Neurosci. 2020;10.1111/pcn.13008. DOI: $10.1111 /$ pcn. 13008

14. Mayland CR, Harding AJ, Preston N, Payne S. Supporting Adults Bereaved Through COVID-19: A Rapid Review of the Impacto Previous Pandemic Songrief and Bereavement. J Pain Symptom Manage. 2020 May 15. DOI: 10.1016/ j.jpainsymman.2020.05.012

15. Carr D, Boerner K, Moorman S. Bereavement in the Time of Coronavirus: Unprecedented Challenges Demand Novel Interventions. J Aging Soc Policy. 2020;1-7. DOI: 10.1080/08959420.2020.1764320

16. Moore KJ, Sampson EL, Kupeli N, Davies N. Supporting families in end-of-life care and bereavement in the COVID-19. Int Psychogeriatrics. 2020 Apr 30;1-4. DOI: 10.1017/S1041610220000745

17. Souza CP, Souza AM. Rituais Fúnebres no Processo do Luto: Significados e Funções. Psic.: Teor. e Pesq. 2019;35:e35412. DOI: https://doi. org/10.1590/0102.3772e35412

18. Kovács MJ, Vaiciunas NA, Elaine GR. Profissionais do Serviço Funerário e a Questão da Morte. Psicol cienc prof. 2014;34(4):940-54. DOI: https://doi. org/10.1590/1982-370001272013

19. Busa ALA, Silva GB, Rocha FP. O Luto do Jovem Adulto Decorrente da Morte dos Pais pelo Câncer. Psicol cienc prof. 2019;39:e183780. DOI: https:// doi.org/10.1590/1982-3703003183780

Recebido: 27 de maio de 2020 Aprovado: 8 de junho de 2020 Publicado: 2 de julho de 2020

A Revista Baiana de Enfermagem utiliza a Licença Creative Commons - Atribuição-NãoComercial 4.0 Internacional. https://creativecommons.org/licenses/by-nc/4.0/ Este artigo é de acesso aberto distribuído sob os termos da Licença Creative Commons (CC BY-NC). Esta licença permite que outros remixem, adaptem e criem a partir do seu trabalho para fins não comerciais. Embora os novos trabalhos tenham de lhe atribuir o devido crédito e não possam ser usados para fins comerciais, os usuários não têm de licenciar esses trabalhos derivados sob os mesmos termos. 